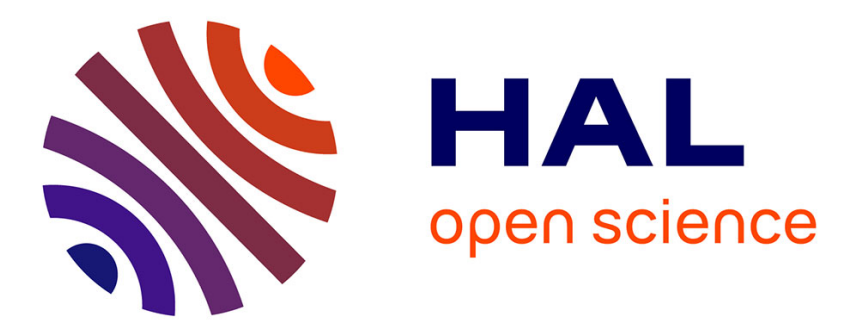

\title{
La déroute militaire comme épreuve mystique: retour sur un passage du Règlement de la guerre, 1QM xvi, 11 - xvii, 9 \\ Christophe Batsch
}

\section{- To cite this version:}

Christophe Batsch. La déroute militaire comme épreuve mystique: retour sur un passage du Règlement de la guerre, 1QM xvi, 11 - xvii, 9. La mystique théorétique et théurgique dans l'antiquité gréco-romaine. Judaïsmes et christianismes, 2016, 978-2-503-56188-2. hal-01670202

\section{HAL Id: hal-01670202 \\ https://hal.univ-lille.fr/hal-01670202}

Submitted on 21 Dec 2017

HAL is a multi-disciplinary open access archive for the deposit and dissemination of scientific research documents, whether they are published or not. The documents may come from teaching and research institutions in France or abroad, or from public or private research centers.
L'archive ouverte pluridisciplinaire HAL, est destinée au dépôt et à la diffusion de documents scientifiques de niveau recherche, publiés ou non, émanant des établissements d'enseignement et de recherche français ou étrangers, des laboratoires publics ou privés. 


\title{
La déroute militaire comme épreuve mystique : retour sur un passage du Règlement de la guerre, 1QM xvi, 11 - xvii, 9
}

\author{
CHRISTOPHE BATSCH \\ Université de Lille
}

\begin{abstract}
Summary
This paper focuses mainly on two points. On the one hand, it tries to demonstrate that the first wars described in the introduction of the War Scroll (col. I), are actual historical events, understood as signs announcing the eschatological war. On the other hand, it interpretates the curious moment when the Sons of Light are temporarily defeated as a final mystical ordeal, which for the members of the yahad are to be prepared.
\end{abstract}

Résumé

Deux points principaux sont développés dans cette contribution. D'une part, on tend à montrer que les premières guerres décrites dans l'introduction du Règlement de la guerre (col. I) correspondent à des événements historiques interprétés comme des annonces de la guerre eschatologique. D'autre part, on tend à comprendre le curieux passage de la défaite provisoire des Fils de Lumière comme une ultime épreuve mystique à laquelle les membres de la Communauté doivent se préparer.

On admet généralement que les Chants de l'holocauste $d u$ shabbat relèvent d'une forme de littérature mystique, dans la mesure où ils attestent le souci d'une célébration terrestre du service divin, parallèle et synchronisée avec le culte accompli aux cieux par les êtres célestes ${ }^{1}$. Si l'on retient cette approche de la mystique juive ancienne, cela devrait nous conduire à nous interroger sur un autre ensemble d'écrits qumrâniens communautaires où s'exprime la même exigence de synchroniser des activités terrestres et célestes : je veux parler du corpus des écrits guerriers de Qumrân ${ }^{2}$.

1. Voir notamment P. S. AlEXANDER, The Mystical Texts. Songs of the Sabbath Sacrifice and Related Manuscripts, Londres, T\&T Clark International, 2006 ; et l'article de David Hamidović, « La contribution des Cantiques de l'holocauste du sabbat à l'étude de la pensée mystique juive au tournant de notre ère », dans ce volume. Il est vrai que certains savants contestent cette interprétation étendue du caractère mystique des Cantiques ainsi E. R. WolfSON, " Mysticism and the Poetic-Liturgical Compositions from Qumrân. A Response to Bilhah Nitzan », The Jewish Quarterly Review 85 (1994), p. 185-202, a-t-il pu considérer que le seul texte véritablement mystique de Qumrân était l'écrit intitulé 4QSelf-Glorification.

2. Que l'on peut distribuer si l'on veut en trois groupes, en suivant la suggestion faite par B. SCHULTZ, Conquering the World. The War Scroll (1QM) Reconsidered, Leiden, Brill, 2009 : (1) Le Règlement de la guerre ou Serekh ha Milhamah(1QM, 4Q492,494,495); (2) Le Livre de la guerre ou Sefer ha Milhamah (4Q285, 11Q14); (3) Les écrits en gestation ou incertains (4Q471, 491, 493, 496). 


\section{Le consensus savant}

Le Règlement de la guerre de la première grotte (1QM) a en effet été complété, au fur et à mesure de la publication des manuscrits, par un petit groupe de textes traitant également des conditions et des caractéristiques d'une guerre eschatologique. Il n'en demeure pas moins le texte communautaire de référence par rapport auquel tous les autres s'étalonnent ${ }^{3}$. C'est d'un passage particulièrement déconcertant de ce Serekh ha Milhamah que je veux traiter ici, en suggérant qu'une lecture «mystique » pourra nous aider à en rendre compte.

En dehors de l'édition de ces nouveaux écrits guerriers, très peu de progrès ont été faits dans la compréhension du Règlement de la guerre depuis la publication en 1977 de l'étude magistrale de Philip Davies ${ }^{4}$. Celui-ci a établi de façon définitive le caractère composite et l'histoire littéraire complexe du texte. Il en a dégagé les différentes strates littéraires et, si certaines de ses conclusions ont pu être remises en cause à la marge, on peut considérer qu'un relatif consensus savant est désormais établi sur les points suivants.

En premier lieu, il s'agit d'un texte composite. L'auteur final a rassemblé et harmonisé plusieurs documents auxquels il a prêté une signification nouvelle en y adjoignant une introduction herméneutique de son propre fonds. On considère généralement que cette introduction correspond à la colonne I.

Ensuite, compte tenu de ce caractère composite, la question de la datation concerne avant tout le moment où cette compilation a été rassemblée et organisée. On s'accorde à considérer que la plus grande partie des documents rassemblés ici date de l'époque hasmonéenne. En revanche, s'agissant de la composition finale, deux hypothèses ont été énoncées : soit aux débuts de la communauté, c'est-à-dire probablement au début de l'époque hasmonéenne; soit au début de la période romaine, c'est-à-dire au début de l'époque hérodienne.

Troisièmement, l'ensemble des spécialistes du Règlement sont unanimes pour considérer qu'il existe un lien étroit entre la première colonne du texte et le document constitué par les colonnes XV à XIX. On est donc en présence de quatre documents harmonisés en un seul texte :

i. Les colonnes II à IX reflètent sans doute un ancien manuel militaire, adapté ensuite aux représentations guerrières et symboliques de la communauté. Ce document principalement tactique développe les éléments d'organisation pratique de l'armée des Fils de Lumière ; il s'inscrit dans le cadre plus général d'un conflit contre l'ensemble des nations.

ii. Les colonnes X à XIV, qu'on peut rattacher au bloc précédent, offrent un recueil liturgique assez hétérogène de prières, discours et exhortations, placés dans la bouche des prêtres à la tête de l'armée. Les différents éléments sont liés par des interpolations dues au compilateur final.

${ }^{3}$. J'entends pour les chercheurs et savants contemporains. Rien ne permet en revanche de décider si l'un ou l'autre des écrits militaires de Qumrân (si oui lequel) faisait plus ou moins autorité au sein de la communauté.

4. Ph. R. DAvies, IQM, the War Scroll from Qumrân. Its Structure and History, Rome, 1977. Analyse reprise et complétée par la suite dans Ph. R. DAVIES, « War of the Sons of Light against the Sons of Darkness », dans L. SchiffMAn - J. VAnderKam (éd.), Encyclopedia of the Dead Sea Scrolls, Oxford-New York, Oxford University Press, 2000, p. 967-968; Ph. R. DAVIES, «The Biblical and Qumranic Concept of War», dans J. Charlesworth (éd.), The Bible and the Dead Sea Scrolls. The Princeton Symposium on the Dead Sea Scrolls, I. Scripture and the Scrolls, Waco/Texas, Baylor University Press, 2006, p. 209-232 ; Ph. R. DAVIES, «Dualism in the Qumran War Texts », dans G. XerAviTs (éd.), Dualism in Qumran, Londres, T\&T Clark, 2010, p. 8-19. 
iii. et iv. Les colonnes XV à XIX ainsi que la colonne I (l'introduction) décrivent une série de combats, finalement victorieux, menés contre des adversaires identifiés comme « les Kittim »; ces combats se déroulent parallèlement sur terre et dans les cieux.

Enfin, tous les chercheurs admettent que le Règlement fait référence à plusieurs conflits distincts dont l'ultime épisode est celui d'une guerre eschatologique - donc encore à venir. Les désaccords sont nombreux en revanche sur le nombre de ces guerres et leur caractère plus ou moins historique. L'étude la plus récente, celle de Brian Schultz, suggère par exemple de distinguer entre deux récits imbriqués décrivant deux conflits distincts lors de la fin des temps ${ }^{5}$. D’une part, la guerre contre les Kittim («War against the Kittim») qui se déroulerait en un temps court et en sept assauts sur la terre d'Israël; d'autre part, la guerre contre les nations («War of the divisions ») qui se déroulerait durant 33 à 40 ans et qui viserait à la conquête du monde. Il faut reconnaître que ce scénario complexe, mais plus ingénieux que convaincant, n'a pas entraîné l'adhésion générale.

Dans ce scénario, l'une et l'autre des ces guerres sont donc des projections dans le futur opérées par les rédacteurs du Règlement, des prophéties inspirées de celles élaborées par le rédacteur de Dn 11-12. Seule la première de ces guerres, celle des sept assauts contre le Kittim, présenterait un caractère plus « réaliste », plus enraciné dans la réalité géopolitique de l'époque, puisqu'elle serait menée contre la puissance séleucide ; la victoire n'en serait pas moins obtenue de façon miraculeuse, grâce à une intervention divine ${ }^{6}$. La seconde guerre est plus hypothétique encore, puisqu'elle est censée mener la communauté à la conquête du monde connu. L'élément fondamental de la lecture de Schultz est donc bien de considérer le Règlement comme un texte prophétique, et les deux guerres qu'il y distingue comme des événements historiques à venir.

Sans m'attarder plus que nécessaire sur cette dimension historiographique du Règlement, j'en suggère pour ma part une lecture toute différente. Je m'efforcerai en effet de montrer ici que la colonne I du manuscrit mentionne trois types de conflits distincts, dont les deux premiers évoquent des événements historiques réels et proches de la date de la rédaction. Ces conflits historiques et vécus, auxquels se réfère l'auteur du texte, ont pour fonction d'annoncer et de préfigurer la guerre eschatologique attendue pour une date proche, principal objet de la compilation. Nous tâcherons d'exposer brièvement quelles sont les guerres historiques (ou plutôt les types de guerres) évoquées dans cette première colonne.

5. B. Schultz, Conquering the World. The War Scroll (1QM) Reconsidered, Leiden, Brill, 2009. À compléter par B. SchUlTZ, « Compositional Layers in the War Scroll (1QM)», dans D. PARRY et al. (éd.), Qumran Cave 1 Revisited. IOQS Ljubliana 2007 Volume, Leiden, Brill, 2010, p. 153-164 ; B. SCHULTZ, « Not Greeks but Romans. Changing Expectations for the Eschatological War in the War Texts from Qumran », dans M. Popović (éd.), The Jewish Revolt Against Rome. Interdisciplinary Perspectives, Leiden, Brill, 2011, p. 107127 ; B. SchultZ, « Re-Imagining the Eschatological War: 4Q285/11Q14 », dans A. MAEIR - J. MAGNESS L. SCHIFFMAN (éd.), "Go out and study the land» (Judges 18:2): Archaeological, Historical, and Textual Studies in Honor of Hanan Eshel, Leiden, Brill, 2012, p. 197-212.

6 . Par exemple, B. Schultz, Conquering the World. The War Scroll (1QM) Reconsidered, Leiden, Brill, 2009, p. 169 : «1QM I describes a war that will be fought when the king of the Kittim, meaning te Seleucid monarch, will decide to launch out from Egypt, which he will have just conquered, on a campaign to the north that will take him through the Land of Israel. In his fury, he will want to completely devastate it. Assisting him will be a number of Judea's neighbors who will have previously aligned themselves with him, as well as some Jews, the "violators of the covenant". Opposing them will be the rest of the Jews from the three tribes who have returned from exile, including but not limited to the sectarians. The fighting will be fierce, even hurried, with seven rounds, each party successively gaining the upper hand, until finally in the seventh round, God's miraculous intervention will give the victory to the Sons of Light. This will allow them to gain control of Jerusalem, marking the end of Israel' spiritual exile. Such a scenario for the eschatological war, inspired by the unfulfilled portion of Daniel's prophecy in ch. 11, was most likely composed early on in the Hasmonean period $»$. 


\section{Des guerres historiques}

Bien qu'elle abonde en précisions géopolitiques, la colonne I ne permet pas de déterminer à quels conflits précis son texte se réfère. Cette apparente contradiction est aisément compréhensible, si l'on songe que la Judée des deux derniers siècles précédant l'ère vulgaire a connu une abondance remarquable de conflits, sur son sol ou à ses frontières. En réalité le texte prétend moins décrire telle guerre en particulier, qu'il ne définit deux catégories de guerres, l'une et l'autre familières aux Judéens de son époque, plusieurs fois renouvelées dans la réalité des événements historiques et probablement ressenties comme des menaces latentes et toujours susceptibles de se reproduire. C'est donc moins à une page d'histoire événementielle que nous avons affaire ici qu'à une typologie des conflits en Judée entre 170 et 60 av.

La première catégorie de ces conflits est présentée aux lignes $1 \mathrm{~b}$ à $3 \mathrm{a}$, c'est-à-dire immédiatement après le titre (ou l'incipit qui en tient lieu) :

1. [...] contre la troupe d'Edom, de Moab et des fils d'Ammon ; 2. puis [...] Philistie ; puis contre les troupes des Kittim d'Assur et leurs alliés, les traîtres à l'Alliance, qui les assistent. Descendants de Lévi, de Juda et de Benjamin, les exilés du désert leur feront la guerre. 3. [...] contre toutes leurs troupes quand reviendront d'exil les fils de Lumière, du désert des peuples pour camper au désert de Jérusalem.

De façon on ne peut plus classique, sont définis ici les deux camps antagonistes et leurs buts de guerre. Les troupes amies sont donc idéalisées dans un récit qui les rattache à l'histoire de la communauté de Qumrân : «Lévi, Juda et Benjamin » représentent les deux royaumes réunifiés sous l'autorité sacerdotale ${ }^{7}$; les « exilés du désert» constituent une référence directe à la théorie historique du « petit reste » qui fonde la légitimité de la Communauté. En revanche, la coalition des ennemis est d'une grande précision géopolitique : on y retrouve les Iduméens et les Transjordaniens sous les termes d' « Edom, Moab, Ammon »; les villes côtières, grecques, nabatéennes ou phéniciennes comme Gaza et Askelon sous l'appellation générale de "Philistie»; le royaume hellénistique des Séleucides et les cités grecques de la périphérie septentrionale de Judée sont désignés comme les « Kittim ${ }^{8}$ d'Assur »; enfin le texte dénonce le parti des Juifs hellénisés et renégats ${ }^{9}$, considérés comme

7. La discussion reste ouverte sur ce point : faut-il rattacher (comme je le fais ici, partageant l'opinion de B. Schultz sur ce point précis) « les fils de Lévi, Juda et Benjamin » au camp des fils de Lumière, ou au contraire au camp des ennemis, comme le fait John Screnock à la suite de bon nombre de commentateurs ? Voir J. SCRENOCK, "Word Order in the War Scroll (1QM) and its Implication for Interpretation », Dead Sea Discoveries 18 (2011), p. 29-44. Dans ce dernier cas, ils pourraient représenter le judaïsme « renégat» des prêtres du Temple de Jérusalem, honnis par la communauté. Cela ne change pas le fond de mon analyse.

${ }^{8}$. Sans revenir sur un débat qui a fait son temps, le fait que le gentilice Kittim désigne ici des Grecs ne signifie pas qu'il en aille toujours ainsi dans la littérature qumrânienne. Je partage tout à fait sur ce point l'analyse de Hanan Eshel concernant «the changing notion of the ennemy » identifé sous ce nom. Voir H. Eshel, "The Kittim in the War Scroll and in the Pesharim », dans D. GoodBlatT - A. PINNICK D. Schwartz (éd.), Historical Perspectives. Jewish Perspectives from the Maccabees to Bar Kochba in Light of the Dead Sea Scrolls. Proceedings of the Fourth International Symposium of the Orion Center, 27-31 January 1999, Leiden, Brill, 2001, p. 29-44.

9 . Concernant cette dernière catégorie, dont on peut penser qu'elle intéressait le plus vivement le rédacteur, un article de John Screnock propose une autre lecture du statut des «descendants de Lévi, de Juda et de Benjamin ». Ce sont également des «traîtres à l'Alliance» (J. SCRENOCK, «Word Order in the War Scroll (1QM) and its Implication for Interpretation », Dead Sea Discoveries 18 (2011), p. 29 : "Word order supports an identification of the sons of Levi, Judah, and Benjamin as "violators of the covenant" » et p. 43 : "They are given a negative connotation (contra Schultz) and are connected to the temple establishment, as Eshel argued »). 
des « traitres à l'Alliance ». Il n'est pas difficile de repérer dans cette énumération la série de conflits au moyen desquels les Hasmonéens sont parvenus à bâtir puis à élargir l'État judéen. On peut pratiquement dresser la liste parallèle des guerres qu'ils ont conduites contre les territoires mentionnés ici. Quant à l'objectif de ces guerres, le contrôle de Jérusalem, il ne va pas sans une certaine ambiguïté : il est indiscutable que les Maccabées, puis les Hasmonéens, ont su s'assurer le contrôle total de Jérusalem pour diriger la Judée ; mais on peut penser aussi que les rédacteurs qumrâniens gardaient à l'esprit leur objectif d'installer au Temple de Jérusalem le «véritable » sacerdoce et le culte authentiquement juif dont ils s'estimaient les représentants, contre la classe sacerdotale en place.

La première vague des conflits annonciateurs de la guerre eschatologique est donc constituée, dans la colonne I du Règlement par l'ensemble des guerres hasmonéennes, en tout cas celles menées depuis Jean Hyrcan jusqu'à Alexandre Jannée. Si l'on adoptait ici la terminologie rabbinique (avant la lettre) on dirait qu'il s'agissait alors de " guerres de librechoix » $(\text { reshut })^{10}$.

La deuxième catégorie de guerres est décrite aux lignes $3 b$ à 7 :

[...] Ils monteront de là-bas 4 [...] les Kittim d'Égypte ; et au temps assigné "il déboulera en grande fureur" (Dn 11,44) pour faire la guerre aux rois du Nord, et sa colère viendra détruire et abattre la corne 5 d'I[sraël [...] Il se produit un trouble 6 im[mense chez] les fils de Japhet, puis Assur tombe sans recevoir aucun secours et la domination des Kittim s'achève.

On a depuis longtemps observé que ce passage s'inspirait assez directement mais librement du chapitre 11 du livre de Daniel, c'est-à-dire de la longue prophétie ex eventu concernant la campagne d'Antiochos IV en Égypte, suivie de son retour à travers la Judée : premier indice évidemment essentiel.

Il est en outre frappant que, dans ce passage du Règlement, les ennemis d'Israël (Kittim, du Nord et d'Égypte, Assur, fils de Japhet) se combattent d'abord entre eux ; c'est seulement en raison et à la suite de ces combats qui ne la concernent pas directement que la Judée (« la corne d'Israël») subit, par ricochet, de grands dommages. Ces antagonistes - dont aucun n'est l'allié des Judéens - sont facilement identifiables : tous Grecs (« Kittim, fils de Japhet », 1. 6), ce sont, d'une part, les Lagides d'Égypte (nommée à la ligne 4) et, d'autre part, les Séleucides de Syrie reconnaissables sous leurs sobriquets de « rois du Nord» et « Assur». Ce sont donc bien les conflits incessants entre les deux puissances hellénistiques qui sont visés ici. Mais pourquoi devrions-nous nous en tenir, comme beaucoup de commentateurs, à la seule figure d'Antiochos IV ? Dans la période assez courte qui nous occupe, on peut repérer au moins trois autres guerres dont la Judée fut le terrain ou la victime: l'expédition de Ptolémée VI Philometor ca 150-145, à l'époque de Jonathan; les guerres de Ptolémée VII Evergète II contre Démétrios II $c a$ 129-125, à l'époque de Jean Hyrcan ; enfin (et sans doute la pire de toutes pour la population judéenne) la fameuse " guerre des sceptres » mettant aux prises Ptolémée IX Soter II, Cléopâtre III et Démétrios III $c a$ 102-88, du temps d'Alexandre Jannée ${ }^{11}$.

Cette deuxième série de guerres ne mettait pas forcément aux prises des troupes judéennes avec leurs ennemis, mais les conséquences pour la population judéenne étaient sans aucun doute bien pires qu'à l'occasion des guerres de conquêtes menées par les Hasmonéens

${ }^{10}$. Par opposition aux « guerres de devoir» (mitswa). Voir notamment Michna Sota VIII.

11. Sur cette dernière guerre, voir E. VAN 'T DACK et al. (éd.), The Judean-Syrian-Egyptian Conflict of 103-101 BC. A Multilingual Dossier Concerning a "War of Scepters", Bruxelles, 1989. Sur les "guerres grecques » de l'époque hasmonéenne, voir H. EsHEL, The Dead Sea Scrolls and the Hasmonean State, Grand Rapids/Michigan, Eerdmans, 2008. 
au-delà de leurs frontières. Les troupes égyptiennes et syriennes qui traversaient la Judée ou s'affrontaient sur son sol, composées de mercenaires et engagées dans ce qui s'apparentait à des guerres civiles, ravageaient sauvagement le pays. Parmi d'autres horreurs, Josèphe mentionne ainsi les pratiques cannibales attribuées un temps aux troupes de Ptolémée Lathyre $^{12}$ en Judée (Ant. Jud. XIII 345-346). C'est évidemment cette dimension horrifique des expéditions militaires des voisins grecs de la Judée qui a motivé leur évocation dans ce texte, comme signe annonciateur de la guerre eschatologique.

La troisième et dernière forme de guerre évoquée dans la colonne I du Règlement concerne en effet cette guerre ultime, non encore advenue mais attendue pour la (très proche) fin des temps.

\section{La guerre eschatologique}

La dimension future de cette dernière forme de guerre est soulignée dans le texte de deux manières. D'une part, au moyen d'un emploi systématique de l'inaccompli. D'autre part, avec des références explicites à des temps non encore advenus : ubayom « et le jour où...» (1. 9.12); ki huh yom ye 'ud lo mé'az «car ce sera le jour fixé par Lui depuis longtemps » (1. 10) ; wehayah 'et « et ce sera un temps...» (1. 11).

À la différence des deux premiers types de guerres, dont la description s'appuyait sur des expériences historiques récentes et vécues, celle qui va être évoquée ici est donc parfaitement imaginaire ou, ce qui revient au même mais dans un autre registre, prophétique. Il serait donc vain de vouloir identifier les entités géopolitiques qui se dissimulent sous les termes symboliques employés (par exemple, à nouveau celui de Kittim), comme nous avons pu le faire jusqu'ici. En revanche, on doit souligner que se manifeste dès lors une dimension nouvelle et absolument essentielle du déroulement de cette guerre entre les forces divines et les forces du mal : elle se déroulera simultanément et synchroniquement sur terre et dans les cieux. Ce lien entre ciel et terre est évoqué ici pour la première fois, car il n'avait évidemment pas sa place à l'occasion des guerres historiques et trop humaines décrites précédemment.

C'est en cela que cette guerre de la fin des temps cesse de ne présenter qu'un caractère eschatologique, en quelque sorte "classique», pour revêtir sous la plume du rédacteur qumrânien une dimension mystique, fondée sur l'étroite union du ciel et de la terre. Comme l'a bien montré Jean Duhaime, « les deux camps participent à un combat surnaturel d'ampleur cosmique. Le lot de la lumière et celui des ténèbres ont à leur tête des puissances célestes accompagnées de milices spirituelles qui combattent aux côtés des humains. Dieu lui-même intervient dans la phase ultime des combats ${ }^{13}$.

Les combats sont menés en commun (1QM I 10-11) :

Et ce (jour-) là la communauté des êtres célestes et le rassemblement des hommes viendront combattre ${ }^{14}$ pour un immense carnage ; fils de Lumière et lot des ténèbres combattront mêlés.

12 . Le surnom généralement employé pour désigner Ptolémée IX Soter II.

13. J. DuhAIME, « La Règle de la guerre (1QM) et la construction de l'identité sectaire », dans F. GARCIA Martinez - M. Popović (éd.), Defining Identities. We, You and the Others in the Dead Sea Scrolls, IOQS Gröningen 2004 Volume, Leiden, Brill, 2008, p. 131-145 (ici 140).

14. יתקרבו Ce hitpe 'el de קרב (forme non attestée dans la Bible hébraïque) peut suggérer une forme de solidarité réciproque : êtres céleste et êtres humains s'avancent simultanément aux combats. 
Autre conséquence importante, la victoire (ou la défaite) sont partagées selon 1QM I 14-15. Principalement, deux lectures succinctes ont été faites de cette phrase.

Pour André Dupont-Sommer, mais il est bien seul, il y est question d'une victoire des fils de Lumière : « La grande Main de Dieu soumettra [Bélial à tou]s les anges de Son empire et à tous les hommes [de Son lot]» ${ }^{15}$. Hommes et anges relèvent ici du lot de Dieu. La majeure partie des traducteurs y repèrent plutôt une évocation de la défaite de Bélial et des siens : « La grande main de Dieu écrasera [Bélial et tou]s les anges de son empire et tous les hommes [de son lot] ». Hommes et anges relèvent alors du lot de Bélial. Dans l'un et l'autre cas, la symbiose des deux composantes, céleste et humaine, des armées qui s'affrontent est soulignée ; dans l'un et l'autre cas sont donc définies ici les prémices d'une potentielle unio mystica.

Cette union mystique est-elle envisagée plus précisément dans ce texte ? Dans quelles circonstances et à quelles conditions ? L'hypothèse que je défendrai ici est qu'un parcours mystique de cette nature est développé à l'occasion de ce curieux passage que Duhaime a qualifié très justement de « dissonance cognitive ${ }^{16}$ » et qui décrit une défaite provisoire des fils de Lumière.

\section{La défaite provisoire}

On a déjà souligné l'unité de pensée et de représentations entre la colonne I, que nous venons d'analyser, et le bloc textuel des colonnes XV-XIX, où figure ce passage sur une défaite momentanée des fils de Lumière. Cette évocation de l'échec, même provisoire, est rare pour ne pas dire inexistante, dans les manuels de guerre anciens. C'est indiscutablement l'une des plus fortes originalités du Règlement. Duhaime en propose cette interprétation finaliste: «Dans la logique proposée ici, le conflit a une force sans précédent parce que le mal doit déployer toutes ses ressources afin d'être éliminé totalement $\gg{ }^{17}$.

Sans contester cette analyse sur le fond, je voudrais observer que le texte du Règlement propose lui-même une interprétation de cette défaite passagère. En effet, après que les combattants provisoirement vaincus se sont repliés, laissant derrière eux un certain nombre de morts, le cohen ha-rosh (le prêtre en chef), c'est-à-dire la plus haute autorité spirituelle et stratégique de l'armée terrestre, leur tient un long discours destiné à rétablir leur moral ${ }^{18}$ en indiquant le sens qu'il convient de donner à cette épreuve.

Deux points sont d'abord établis, qui contribuent à définir ce moment douloureux et incompréhensible (1QM XVI 11) ${ }^{19}$ :

Quand [Bélial] s'armera pour soutenir les fils des ténèbres et que des morts parmi les combattants commenceront à tomber en vertu des mystères divins.

15 . A. Dupont-Sommer, « Règlement de la guerre », dans A. DuPOnT-SOMmER - M. Philonenko (éd.), La Bible. Écrits intertestamentaires, Paris, Gallimard, 1987, p. 194.

16. J. Duhaime, «La Règle de la guerre (1QM) et la construction de l'identité sectaire », p. 143. Le passage en question est celui indiqué dans le titre de cette contribution : 1QM XVI 11 à XVII 9. Les passages parallèles dans les manuscrits de la quatrième grotte (4QM) figurent en 4Q491 10 2,11-14; et 4Q493 2 9. 7. 8-9

17. J. DuHAIME, « La Règle de la guerre (1QM) et la construction de l'identité sectaire », p. 145.

18. Littéralement wehizaq 'et lebabam ainsi il fortifiera leurs cœurs » (1QM XVI 13-14). Cela l'apparente au modèle classique des harangues guerrières dans le judaïsme ancien, dont il se distingue cependant par plusieurs points.

19. Le texte, en partie lacunaire pose des problèmes de lecture. J'adopte celle qui est la plus largement admise et qui restitue «Bélial» dans la lacune. Voir en particulier l'édition la plus récente du Règlement par E. QIMron, The Dead Sea Scrolls. The Hebrew Writtings, I, Jérusalem, Yad Ben-Zvi Press, 2010, p. 109-136. 
Donc :

Cette défaite passagère relève du domaine des razé 'El « les mystères divins »), 1QM XVI, 11 : «Des morts parmi les combattants commenceront à tomber en vertu des mystères divins $^{20} \gg$ etc.

Elle découle de l'intervention d'une puissance spirituelle de l'au-delà (en l'occurrence malfaisante), 1QM XVI, 11 : «Quand [Bélial] s'armera pour soutenir les fils des ténèbres » etc.

Rony Yishai a défini assez justement tout ce discours du prêtre en chef comme une « cérémonie prédéterministe ${ }^{21} »$. La prédétermination demeure en effet un élément essentiel de la doctrine qumrânienne dont on retrouve ici l'expression avec la métaphore du « creuset » (masref) qui structure l'introduction du discours.

Mais il convient d'aller plus loin. Le cohen ha-rosh développe en effet ici une harangue en sept points, assez éloignée du modèle classique de la harangue guerrière sacerdotale, dont le modèle biblique figure en Dt 20,2-4 ${ }^{22}$. Les deux premiers points élaborent une explication vraisemblable, et en effet fondée sur la théorie de la prédestination, au scandale théologique de la mort de certains des combattants de l'armée des fils de Lumière. En revanche, les quatre points suivants développent la représentation d'un parcours aboutissant à une unio mystica avec les puissances spirituelles (bonnes comme malfaisantes), parcours mystique dont le combat lui-même constitue le lieu et le chemin, comme il est indiqué dans la conclusion.

Reprenons point par point le développement de ce discours (1QM XVI 15-XVII 9)

\section{Structure de la harangue du cohen ha-rosh}

\section{L'épreuve et le creuset (1QM XVI 15-XVII 1)}

Comment comprendre la mort au combat de certains des fils de Lumière ? A priori ils appartenaient au monde des justes. L'explication offerte ici fait appel au prédéterminisme individuel qumrânien. On sait comme le syntagme «mystères divins » (razé 'El) exprime essentiellement, à Qumrân, cette connaissance de la théorie de la prédestination ${ }^{23}$. Donc, même au sein des justes, il subsiste encore quelques parts de ténèbres. Le combat contre Bélial devient alors le «creuset» au fond duquel Dieu «a fait l'épreuve du cœur de son peuple ». Puisque certains parmi les fils de Lumière y ont été brûlés, il s'ensuit qu'ils devaient l'être: en d'autres termes leur destinée, déterminée dès leur naissance (voire dès leur conception) et probablement calculable en parts de lumière et en parts de ténèbres, ne pouvait

20. Sur cette notion de «mystères divins » à Qumrân, voir notamment V. TRIPLET-HitOTO, Mystères et connaissances cachées à Qumrân. Dt 29,28 à la lumière des manuscrits de la mer Morte, Paris, Cerf, 2011.

21. R. YISHAY, "The Model of the Eschatological War Descriptions in Qumran Literature », dans Meghillot. Studies in the Dead Sea Scrolls 4 (2006), p. 121-139 (en hébreu).

${ }^{22}$. Voir, sur ce texte biblique, l'ouvrage déjà ancien mais toujours pertinent de E. W. CONRAD, Fear not Warrior. A Study of the 'al tira' Pericopes in the Hebrew Scriptures, Chico/Californie, Scholars Press, 1985 ; ainsi que R. SETIO, "The Text of War in the Context of War. A Functional Reading », dans A. HUNTER P. DAVIES (éd.), Sense and Sensitivity: Essays on Reading the Bible in Memory of Robert Carroll, Londres, Sheffield Academic Press, 2002, p. 289-301.

23. Voir V. Triplet-Hitoto, Mystères et connaissances cachées à Qumrân. Dt 29,28 à la lumière des manuscrits de la mer Morte, Paris, Cerf, 2011. 
leur permettre de survivre à cette épreuve. Raisonnement circulaire mais imparable : le fait même qu'ils aient succombé offre la meilleure preuve qu'ils étaient destinés à succomber.

En sens inverse, les survivants ont acquis la preuve qu'ils étaient destinés à survivre, y compris dans les combats à venir: «ceux qui ont été éprouvés dans le creuset», Dieu «assurera leur intégrité dans les flammes » ${ }^{24}$.

\section{Le modèle biblique : les fils d'Aaron (1QM XVII 2-3)}

L'orateur appuie sa démonstration d'un exemple biblique, tiré du Lévitique. C'est au demeurant la seule anecdote narrative figurant dans ce livre de lois ( $\operatorname{lv} 10,1-3)$ : Aaron a quatre fils, tous destinés à la prêtrise par leur naissance. Deux de ces fils, Nadab et Abihu, accomplissent un rituel incorrect et sont immédiatement châtiés par un feu divin qui les consume : «ils moururent devant YHWH». Aaron et les deux frères survivants doivent s'abstenir de toute forme de deuil et poursuivre le service du Temple.

Le point que souligne l'orateur de 1QM est que les deux frères sont morts en dépit de leur appartenance au groupe élu des prêtres, et que ces morts participaient à la sanctification et à la gloire de Dieu. 1QM XVII 2: "Souvenez-vous du jugement de Nadab et Abihu fils d'Aaron par le jugement desquels Dieu a dévoilé sa sainteté à la face [de tout le peuple ». Il ne fait là que reprendre le vocabulaire du Lévitique (Lv 10,3) : «C'est ce que YHWH a déclaré en ces termes : par ceux qui m'ont approché (i.e. les deux frères fautifs) je serai sanctifié et à la face de tout le peuple je serai glorifié ».

D'où l'on doit conclure : d'abord qu'au sein d'un groupe si fidèle et restreint soit-il, il reste toujours la possibilité d'une épuration plus stricte des élus. Ensuite que ces morts ne sont pas inutiles : elles contribuent à leur façon à la gloire divine ; même les non-parfaits assument ainsi un rôle positif dans les plans divins. Et finalement, par ce biais de la participation à la gloire divine, est introduit le thème de l'union mystique entre les mortels et les puissances spirituelles.

\section{La mystique négative des fils des ténèbres (1QM XVII 4)}

Le cohen ha-rosh revient alors à la structure classique de la harangue, qui fait se succéder l'exhortation à ne pas craindre et à être fort, puis la désignation des ennemis et de leur faute. Mais il inscrit cette forme classique dans le cadre d'une approche mystique. En effet, le reproche formulé ici contre les fils des ténèbres est d'aspirer à se fondre dans le désordre primitif antérieur à la création, l'effrayant tohu et bohu que décrit la Genèse 1,2 :

Or la terre était tohu et bohu avec des ténèbres à la surface du néant.

Selon le texte de la harangue, ce tohu et bohu constitue l'aspiration ultime des ennemis :

${ }^{24}$. On trouve une image proche et analogue dans la longue harangue sacerdotale, de facture plus classique, qui figure aux colonnes X-XII. Le prêtre y prononce (1QM XI 10-11) : « et ceux à l'esprit abbatu (Es. 66,2), Tu (les) mettras au feu comme une torche enflammée dans la paille (Za 12,6) dévorant l'impiété, ne refluant pas avant l'extermination du péché ». Ici aussi, il est possible que certains des combattants parmi les fils de Lumière soient visés ; mais le contexte de ce premier discours est très différent, qui met surtout l'accent sur l'espérance messianique: citation de $\mathrm{Nb} 24,17-19$ (l'étoile de Jacob et le sceptre d'Israël); mention explicite des meshihékah, les oints de Dieu. Aucune trace, en revanche, de prédéterminisme individuel, ni de fusion mystique, en dépit du fait que les annonces prophétiques de la victoire finale soient réitérées. 
Eux c'est au tohu et bohu (que va) leur désir profond.

On doit y reconnaître la représentation d'une forme d'aspiration mystique des fils des ténèbres, par laquelle les êtres humains malfaisants manifestent l'ambition ultime de retourner à un chaos cosmique primitif, au sein duquel ils partageront la nature supraterrestre des puissances du mal.

\section{La mystique positive : l'intervention céleste ici-bas (1QM XVII 5-7)}

À cette mystique du mal, le cohen ha-rosh oppose alors, en la détaillant, les promesses faites aux fils de Lumière d'une mystique positive. La première manifestation en sera l'intervention ici-bas d'une puissance céleste. Non seulement ce rapprochement sans médiation du ciel et de la terre ne causera aucun désordre, mais il contribuera à l'inverse au renforcement de l'Alliance. Cette intervention du «secours décisif» de la puissance spirituelle devra s'opérer sous la forme de « la puissance de l'archange (littéralement « l'ange de majesté ») ${ }^{25}$, en vue de la domination de Michel ».

\section{La mystique positive : le partage des épreuves (1QM XVII 7-8)}

L'harmonie efficace de cette union du ciel et de la terre, dans la lutte contre le mal, se vérifiera dans la répartition des tâches et des épreuves qu'affronteront côte-à-côte les puissances célestes et les armées humaines qui constituent ensemble « le lot, ou le parti, de Dieu » (goral 'El). Tandis qu'à l'archange Michel est réservée la "souveraineté sur les êtres célestes » (be'élim misrat Mikael), à Israël reviendra la "domination sur toutes chairs » (memshalat Israel bekol basar). Cette répartition exprime plus qu'une forme de division du travail : elle garantit le maintien du bon ordre ontologique du monde et le maintien de la distinction entre les univers céleste et terrestre, jusque dans la mise en œuvre de cette unio mystica des justes pour la victoire sur le mal.

\section{La mystique positive : la récompense (1QM XVII 8).}

En dépit du maintien de cette coupure ontologique, les bénéficiaires humains de cette aventure mystique en retireront un profit certain. Dans une communauté qui accorde la primauté à la connaissance des plans ou mystères divins, et qui fonde son existence sur l'accès à cette connaissance que la révélation a autorisé son fondateur à atteindre, il n'est pas plus grande récompense que celle-ci : les êtres humains soumis à ces épreuves et pris dans l'alliance du ciel et de la terre accéderont à la vérité et "jouiront (ou exulteront) de la connaissance éternelle ».

Je n'entre pas ici dans le débat qui vise à déterminer si cette forme d'eschaton implique ou non l'accès à un autre monde et/ou à une forme de vie éternelle, car le texte n'en

25. Sur le rôle nouveau des anges combattants à la fin de l'époque du deuxième Temple, voir A. MichalaK, Angels as Warriors in Late Second Temple Jewish Literature, Tübingen, Mohr Siebeck, 2012. 
parle pas explicitement. Quoi que cela puisse impliquer par ailleurs, le plus haut degré d'expérience mystique semble ici atteint avec la promesse d'un accès à la connaissance parfaite.

\section{La conclusion de la harangue (1QM XVII 8-9).}

La conclusion de la harangue réconfortante du cohen ha-rosh marque un retour aux réalités immédiates : son ultime exhortation aux «fils de son l'alliance » (i.e. de Dieu), est un rappel du fait que le parcours mystique qu'il vient de promettre, et l'épreuve des combats (y compris les moments de défaite passagère), constituent un seul et même moment. D'où, reprenant les termes de son ouverture, son appel à «rester fort dans le creuset de Dieu » (XVII 8), c'est-à-dire à s'aventurer au cœur des combats les plus risqués. Car c'est précisément et uniquement dans ce «creuset» qu'opèrera l'aventure de l'union mystique, lorsque Dieu « emplira ces creusets de ses mystères au sujet de vos existences » (XVII 9).

\section{Conclusion}

Si cette lecture du passage de la défaite passagère est juste, il faudra alors admettre que le Règlement de la guerre fut peut-être l'objet d'une certaine diversité d'interprétations dès l'époque de la communauté de Qumrân. En effet, si son caractère eschatologique et messianique paraît bien établi en plusieurs autres passages, rien en revanche n'interdirait de penser qu'il ait pu être fait de sa lecture un usage d'exercice mystique, quand ce ne serait qu'au titre d'un entraînement aux épreuves des derniers jours. 